\title{
Antioxidant and Anti-inflammatory Activity Screening of Lasia spinosa Rhizome and its Validation using a Computational Simulation Approach
}

\author{
Arya Lakshmi Marisetti ${ }^{1, \star}$, Mohan Gandhi Bonthu ${ }^{2}$, Ganga Rao Battu ${ }^{1}$ \\ ${ }^{1}$ Pharmacognosy and Phytochemistry Research Division, University College of Pharmaceutical Sciences, Andhra University, \\ Visakhapatnam, Andhra Pradesh, India. \\ 2Department of Pharmaceutical Analysis, Sri Vasavi Institute of Pharmaceutical Sciences, Tadepalliguem, Andhra Pradesh, INDIA.
}

\begin{abstract}
Background: The present work assesses the antioxidant and anti-inflammatory effect of Lasia spinosa rhizome extracts on in-vitro and in-vivo models compiled through molecular docking study of plant-steemed phytocompounds with specific targets. Materials and Methods: In this study, Lasia spinosa rhizome was subjected to extraction using petroleum ether, ethyl acetate and methanol and the extracts were analyzed by GC-MS. Antioxidant was assessed using in-vitro methods such as DPPH scavenging activity and $\mathrm{H}_{2} \mathrm{O}_{2}$ scavenging activity; anti-inflammatory activity was assessed using both in-vitro and in-vivo and molecular docking utilizing Auto dock 4.0 was done. Results: Tests showed that methanolic extract (MELS) has the most important dose-dependent antioxidant and anti-inflammatory efficacy at various levels. Of all compounds, Morin reported the most successful docking ranking of -8.2 to -9.8 , maintaining a good binding fondness between protein and ligand. Conclusion: Antioxidant and anti-inflammatory of Lasia spinosa may be inferred from the examinations. The in-vitro, in-vivo and in silico assays of L. spinosa. Morin is confirmed by the information as a beneficial antioxidant and anti-inflammatory agent that can aid future clinical assessments.
\end{abstract}

Key words: Lasia spinosa, Antioxidant, Anti-inflammatory, In silico, Auto dock.

\section{INTRODUCTION}

The natural reaction to external threats such as bacteria, viruses and parasites is inflammation. ${ }^{1}$ Inflammation study is one of the major fields of public health science ${ }^{2}$ owing to the sideeffects of widely used NSAIDs such as renal suppression and gastrointestinal disorders like ulcers. Arachidonic acid conversion of prostaglandin (PGs) involves cyclooxygenase-1 (COX-1 and cyclooxygenase-2 (COX-2, respectively). COX-2 is not usually present and is elevated by carcinogenic or pro-inflammatory cytokines in human tissues. ${ }^{3}$ Latest studies indicate that natural compounds suppress inflammation through selective COX-2 inhibition. ${ }^{4}$
Plants are known as a biologically active chemicals source and for antioxidant and anti-inflammatory function plants have been studied. Lasia spinosa have been used in folk medicine since ancient times, owing to antiinflammatory, anthelmintic, emenogouge, antioxidant, anti-diabetic, antimalarial activities. ${ }^{5,6}$ The present research aims to predict bioactive compounds in methanol extract from L. spinosa by GC-MS study. Methanolic crude Lasia spinosa extract's (MELS) anti-inflammatory behavior and antioxidant function was studied and tested using a numerical simulation methodology. The ADMET property and bioactivity score of the compound was checked to evaluate drug-like characteristics of chosen substances. Docking aims to integrate active
Submission Date: 11-05-2020; Revision Date: 17-07-2020; Accepted Date: 26-10-2020

DOI: 10.5530/ijper.54.4.207 Correspondence:

Mrs. Arya Lakshmi Marisetti Research Scholar, Pharmacognosy and Phytochemistry Research Division, University

College of Pharmaceutical Sciences, Andhra University, Visakhapatnam -530003, Andhra Pradesh, INDIA. Phone no: +919492870889 Email id: maryalakshmi@ gmail.com

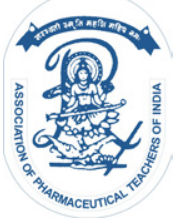

www.ijper.org 
phyto-compounds with oxidation and inflammationenzymes such as cytochrome P450, lypoxygenase, myeloperoxidase, xanthine oxidase and active COX-2 sites. This methodology indicates a systemic framework for potential drug design utilizing active plant metabolites.

\section{MATERIALS AND METHODS}

\section{Plant Material Collection and Storage}

The rhizome of Lasia spinosa were collected from Kommulamamidi, near Kottapalli village, Paderu in Visakhapatnam (Andhra Pradesh) in NovemberDecember 2018. Prof S.B. Padal authenticated the plant materials, Department of Botany (Voucher No: 23302), Andhra University, Visakhapatnam. The collected plants were separated and its rhizome parts are processed. The collected rhizome was sliced and dried under shade for 10 days, coarsely powdered and passed through sieve no. 40 and stored in an airtight container for further study.

\section{Extraction of Plant material and GC-MS analysis}

A $4000 \mathrm{~mL}$ solvent was packed with $1000 \mathrm{~g}$ of dried plant material. A serial non-polar to polar phytochemicals extraction was subsequently conducted utilizing petroleum ether, ethyl acetate and methanol by maceration. By the use of a $60^{\circ} \mathrm{C}$ rotary evaporator methanol extract was concentrated under vacuum.

\section{Phytochemical Analysis}

The different extracts of $L$. spinosa underwent initial phytochemical testing for the recognition of chemical components according to the standard operating procedures. ${ }^{78}$

\section{Antioxidant activity}

\section{DPPH Assay}

A slightly modified 1.1-diphenyl-2-picrylhydrazyl $(\mathrm{DPPH})$ assay $^{9}$ calculated the free radical scavenging activity of each extract. New methanolic DPPH solution $(0.2 \mathrm{mM})$ in the dark $2 \mathrm{hr}$ before examination has been packed and incubated. In their respective extraction solvent, crude extract and even normal ascorbic acid have been dissolved independently. The $0.05 \mathrm{ml}$ of each solution from each sample was then moved to the 96-well plates at varying concentrations. The methanol solution of DPPH $(0.195 \mathrm{ml})$ was added to any well through the use of a multichannel pipette. After $1 \mathrm{hr}$ of dark incubation, the absorption of the resulting mixture was measured with a micro plate reading of $540 \mathrm{~nm}$. DPPH Radical scavenging activity was calculated using the following equation as as the ascorbic acid equivalent by gram dry weight (mg TE / g dry weight):

\section{DPPH radical scavenging activity $=$ Absorbance $_{\text {blank }^{-}}-$ Absorbance $_{\text {sample }} /$ Absorbance $_{\text {blank }} \times 100$}

\section{Hydrogen peroxide Assay}

Dehpour's updated approach calculated the extract behavior for scavenging in relation to hydrogen peroxide radicals. The solution for hydrogen peroxide (40Mm) was made using the $\mathrm{pH} 7.4$ phosphate buffer and its amount was measured using a UV spectrophotometer to determine absorbance at $560 \mathrm{~nm}$. Add $0.1 \mathrm{mg} / \mathrm{ml}$ of the extract and absorption, determined by using a UV spectrophotometer at $560 \mathrm{~nm}$, to a blank solution with a hydrogen peroxide free phosphate tampon. The percentage of hydrogen peroxide scavenging extract and normal compound was determined with the formula:

Hydrogen peroxide scavenging activity $=$ Absorbance $_{\text {blank }}-$ Absorbance $_{\text {sample }} /$ Absorbance $_{\text {blank }} \times$ 100

\section{Identification of the phyto-compound through GC-MS analysis}

The GC-MS analysis was conducted for the purpose of profiling the pharmacologically active portion present in methanol extract. Initial temperatures of $70 \hat{\mathrm{A}}^{\circ} \mathrm{C}$ were sustained for $2 \mathrm{~min}$, elevated higher at $6^{\circ} \mathrm{C} / \mathrm{min}$, to $260^{\circ} \mathrm{C}$ and held for $9 \mathrm{~min}$. As a carrier gas, helium was used and a flow rate of $1 \mathrm{~mL} /$ min was attained. The volume of samples extracted contained $1 \mu \mathrm{L}$ of extract. $^{7}$ A reference for the calculation of important peaks in mass spectrometry was screened in the Library of NIST.

\section{Animals}

The Animal House of Andhra University, Visakhapatnam, were the source of the Wistar rats (100-150 g). It is kept in polypropylene containers, feeding the rodent with a daily diet of about $24 \pm 2$ centimeters in water, $12 \mathrm{hr}$ in light / night, 35-60\% moisture and adds libitum content. No food but water deprived animals $4 \mathrm{hr}$ before the experiment.

\section{Acute toxicity studies}

Anti-inflammatory activity screening of MELS

\section{In vitro Model}

\section{Membrane Stabilization Assay}

The anti-inflammatory function of various Lasia spinosa extracts. The HRBC membrane stabilization process for in vitro $L$. spinosa has been evaluated. 
Blood was collected from rats and has been blended and centrifuged to isosaline in the same amount of Alsever. The same concentration of the research drug at three separate amounts of 100, 200 and $300 \mu \mathrm{g} /$ $\mathrm{mL}$ has been applied to $1 \mathrm{~mL}$ of HRBC suspension. Both research blends were incubated and centrifuged at $37^{\circ} \mathrm{C}$ for $30 \mathrm{~min}$. By using the spectrophotometer at $560 \mathrm{~nm}$, the hemoglobin content of the solution was estimated. The percentage of hemolysis was measured using a spectrophotometer of $560 \mathrm{~nm}^{10}$ as seen below hemoglobin content of the supernatant solution. The hemolysis percentage was then determined according to the following formula:

$\%$ of protection $=100-(\mathrm{OD}$ of test $/ \mathrm{OD}$ of control $)$
$\times 100$

Here "OD of test" is optical density or the test sample's absorbance and "OD of control" is optical density or absorbance of the negative control.

Therefore, Alsever's blood solution and no aspirin or methanol extract of the plant substance are used as a negative control in this event.

\section{In vivo Model}

\section{Carrageenan Induced Paw edema}

The mechanism for the in vivo, anti-inflammatory behavior of methanol extract has been used for Carrageenan-caused rat paw edema. ${ }^{11}$ The rats were divided into 5 classes $(n=6)$, each receiving distilled water (control), 50,100, $200 \mathrm{mg} / \mathrm{kg}$ of MELS and diclofenac of $20 \mathrm{mg} / \mathrm{kg}$ of p.o as reference standard, respectively. In the right hind-paw subplantary tissue of each rodent, carrageenan ( $0,1 \mathrm{~mL}$ of 1 percent) had been injected. The injection volume of carrageen into the foot was measured with a plethysmometer for 0,30 , 60, 120 and $180 \mathrm{~min}$. The inhibition percentage (PI) was calculated at each interval. ${ }^{12}$

$$
\mathrm{PI}=\frac{\left(\mathrm{V}_{\mathrm{t}}-\mathrm{T}_{0}\right) \text { control }-\left(\mathrm{V}_{\mathrm{t}}-\mathrm{V}_{0}\right) \text { treated }}{\left(\mathrm{V}_{\mathrm{t}}-\mathrm{V}_{0}\right) \text { control }} \times 100
$$

$\mathrm{V}_{0}=$ Mean paw volume at $0 \mathrm{hrs}$

$\mathrm{Vt}=$ Mean paw volume at a particular time interval

\section{Statistical Analysis}

All information was revealed as the mean \pm S.D; information went through one-way ANOVA adhered to by Turkey examination. The analytical evaluation executed with Graphpad Prism (Version 3, U.S.A.) software program. $P<0.05$ was taken into consideration statistically considerable.

\section{Molecular Docking}

The docking studies of compounds 3-Carene, 4-hydroxy benzoic acid, Alpha pinene, Apigenin, Camphor, Cinnamic acid, Genistic acid., Morin and Syringic acid were carried out using Autodock 4.0 and Discovery studio Biovia 2019 software to find out the interaction between ligand and the target protein. The crystal structure of enzymes (receptors) that react during metabolism to the development of reactive oxygen species (ROS) are cytochrome P450 (PDB ID: 1OG5), lypoxygenase (PDB ID: 1N8Q), myeloperoxidase (PDB ID: 1DNU) and xanthine oxidase (PDB ID: 3NRZ) was derived from the protein data bank, along with pro inflammatory gene i.e., COX-2 (PDB ID: 5IKR).

\section{ADMET Screening}

ADMET of the ligands is pharmacokinetic properties calculation that is required to be examined to establish their function inside the body. The ADMET inheritance of the ligands was studied, making use of admetSAR. ${ }^{13}$

\section{RESULTS}

\section{Phytochemical Screening of the extract}

Initial phytochemical testing of $L$. spinosa exposed different phytoconstituents detailed in Table 1.

\section{Antioxidant activity}

Extracts were tested with DPPH free radical scavenging and $\mathrm{H}_{2} \mathrm{O}_{2}$ radical scavenging tests for their potential antioxidant function.

\section{DPPH free radical scavenging assay}

DPPH is a stable free radical which shows maximum ultraviolet and visible absorbance at $517 \mathrm{~nm}$. It gets reduced in presence of antioxidant present in the sample which is considered as a measure of their antioxidant activity. The ability of samples to scavenge DPPH radical was measured on the bases of their concentrations providing $50 \%$ inhibition $\left(\mathrm{IC}_{50}\right)$. The results of one way ANOVA test and post hoc test indicates significant difference of mean percentage scavenging between different concentrations of tested extracts. The scavenging activity of extract was well pronounced at higher concentrations of $80 \mathrm{mg} / \mathrm{ml}$ and $100 \mathrm{mg} / \mathrm{ml}$ with a mean percentage of $50.89 \pm 0.58$ and $56.22 \pm 1.41$ for MELS respectively, which was lower, compared with standard antioxidant, ascorbic acid but exhibits similar pattern of concentration dependent free radical 


\begin{tabular}{|c|c|c|c|c|}
\hline Phytoconstituents & Method & $\begin{array}{c}\text { Methanol } \\
\text { Extract }\end{array}$ & $\begin{array}{c}\text { Ethyl acetate } \\
\text { Extract }\end{array}$ & $\begin{array}{c}\text { Pet. Ether } \\
\text { Extract }\end{array}$ \\
\hline \multirow{3}{*}{ Flavonoids } & Shinoda Test & + & + & - \\
\hline & Zn. Hydrochloride test & + & + & - \\
\hline & Lead acetate Test & + & + & - \\
\hline Volatile oil & Stain test & + & - & + \\
\hline \multirow{2}{*}{ Alkaloids } & Wagner Test & + & - & - \\
\hline & Hager's Test & + & - & - \\
\hline \multirow{2}{*}{ Tannins and Phenols } & $\mathrm{FeCl}_{3}$ Test & + & + & - \\
\hline & Potassium dichromate test & + & + & - \\
\hline Saponins & Foaming Test & + & - & - \\
\hline Steroids & Salkowski test & + & - & + \\
\hline Carbohydrates & Molish test & + & - & - \\
\hline Acid compounds & Litmus test & - & - & - \\
\hline Glycoside & Keller-Killani Test & + & - & - \\
\hline Amino acids & Ninhydrin test & + & - & - \\
\hline Proteins & Biuret test & + & - & - \\
\hline
\end{tabular}

"+"-Present; "-"- Absent

\begin{tabular}{|c|c|c|c|c|c|c|c|}
\hline \multirow{2}{*}{ Method } & \multirow{2}{*}{ Treatment } & \multicolumn{5}{|c|}{ Absorbance wavelength (nm) } & \multirow{2}{*}{$I C_{50}$} \\
\hline & & 20 & 40 & 60 & 80 & 100 & \\
\hline \multirow{4}{*}{$\begin{array}{c}\text { DPPH free radical } \\
\text { scavenging (\% Inhibition) }\end{array}$} & PELS & $10.52 \pm 1.16$ & $18.15 \pm 0.55$ & $25.85 \pm 1.21$ & $31.42 \pm 0.64$ & $35.54 \pm 0.12$ & 141.17 \\
\hline & EALS & $18.55 \pm 0.58$ & $28.95 \pm 1.22$ & $36.52 \pm 0.89$ & $45.12 \pm 1.15$ & $51.78 \pm 0.84$ & 93.42 \\
\hline & MELS & $24.55 \pm 0.11$ & $35.82 \pm 1.52$ & $43.82 \pm 2.52$ & $50.89 \pm 0.58$ & $56.22 \pm 1.41$ & 79.73 \\
\hline & Ascorbic acid & $31.21 \pm 1.44$ & $44.32 \pm 1.82$ & $61.17 \pm 0.44$ & $69.24 \pm 0.25$ & $81.12 \pm 1.25$ & 16.06 \\
\hline \multirow{4}{*}{$\begin{array}{c}\mathrm{H}_{2} \mathrm{O}_{2} \text { scavenging activity (\% } \\
\text { Inhibition) }\end{array}$} & PELS & $12.52 \pm 0.75$ & $18.12 \pm 0.52$ & $25.78 \pm 0.15$ & $29.55 \pm 0.52$ & $35.11 \pm 0.88$ & 141.78 \\
\hline & EALS & $24.75 \pm 1.52$ & $28.52 \pm 0.38$ & $34.22 \pm 0.69$ & $39.41 \pm 0.21$ & $42.58 \pm 0.41$ & 93.42 \\
\hline & MELS & $30.22 \pm 0.58$ & $41.82 \pm 0.05$ & $45.63 \pm 0.15$ & $53.66 \pm 1.18$ & $61.12 \pm 0.08$ & 79.73 \\
\hline & Ascorbic acid & $48.89 \pm 0.68$ & $54.58 \pm 0.47$ & $68.67 \pm 0.52$ & $76.22 \pm 0.41$ & $88.12 \pm 1.14$ & 28.52 \\
\hline
\end{tabular}

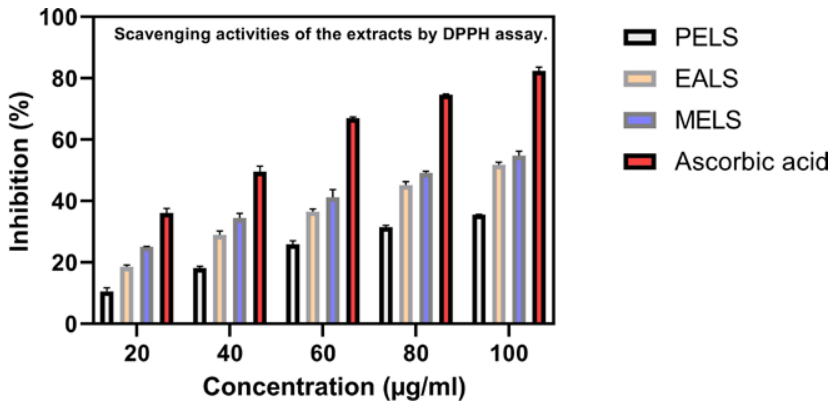

Figure 1: Scavenging activities of the extracts by DPPH assay. The results are expressed as mean $\pm \operatorname{SD}(n=3)$. scavenging effect. The $\mathrm{IC}_{50}$ values of PELS (151.06), EALS (129.16), MELS (69.53) and ascorbic acid (28.52) were obtained using the linear regression equation. The radical scavenging ability, $\mathrm{IC}_{50}$ of extracts and ascorbic acid were presented in Table 2 and Figure 1.

\section{$\mathrm{H}_{2} \mathrm{O}_{2}$ radical scavenging assay}

Table 2 demonstrates the extract's scavenging potential on $\mathrm{H}_{2} \mathrm{O}_{2}$. The extract showed the dose-dependent behavior of hydrogen peroxide radical scavenging. In contrast with other samples, hydrogen peroxide radical 


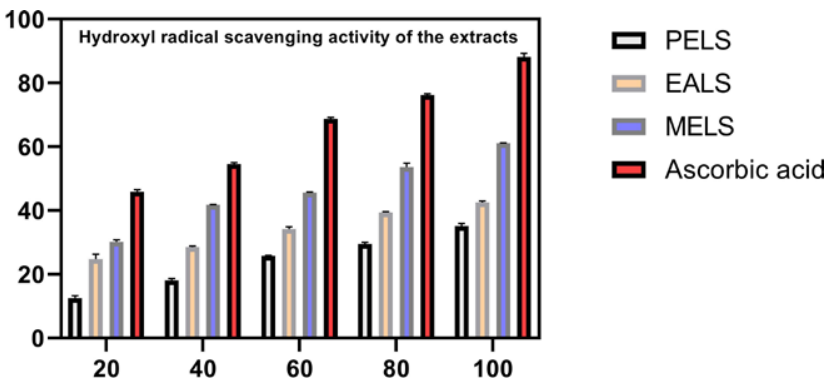

Figure 2: Scavenging activities of the extracts by $\mathrm{H}_{2} \mathrm{O}_{2}$ assay. The results are expressed as mean $\pm \mathrm{SD}(n=3)$.

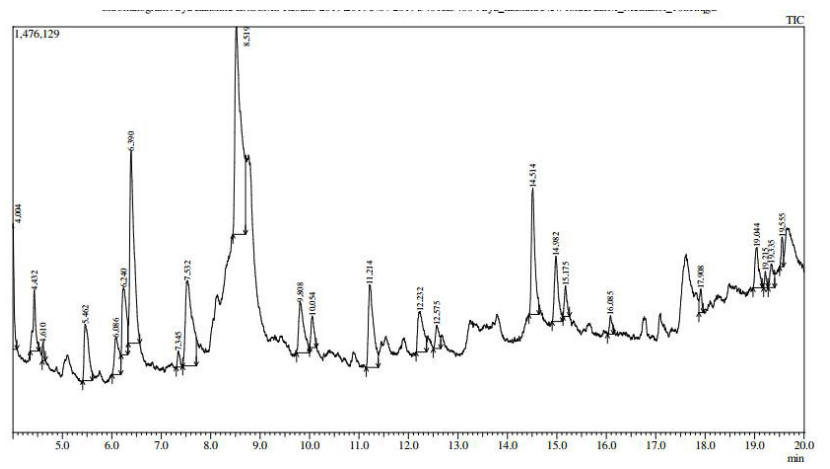

Figure 3: GC Spectra of Lasia spinosa methanol crude extract.

scavenging behaviors of MELS were significant at all concentrations. Similar dose dependent scavenging was observed for standard, ascorbic acid. The $\mathrm{IC}_{50}$ value of the extract was $69.53 \mathrm{mg} / \mathrm{ml}$ whereas for ascorbic acid it was $28.52 \mathrm{mg} / \mathrm{ml}$ using linear regression equation (Figure 2).

\section{GC-MS analysis}

The amount of methanol extract yield was $8.3 \%$. Figure 3 reveals the main peak found in the methanol sample and the NIST Library was referred to classify each compound based on the peak area. Table 3 demonstrates bioactive compounds in Lasia spinosa methanol extract. Based on the GC-MS spectra, the

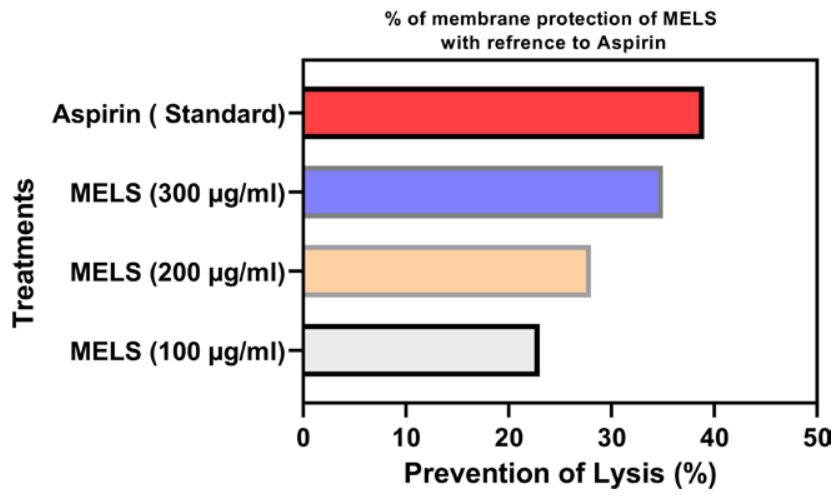

Figure 4: The bar chart above represents the percentage of membrane protection of the MELS with a reference to Aspirin $100 \mu \mathrm{g} / \mathrm{mL}$ as standard.

active compounds in the extract were classified as 3-Carene, 4-Hydroxy Benzoic Acid, $\alpha$-Pinene, Apigenin, Camphor, Cinnamic Acid, Genistic Acid, Morin and Syringic acid (CAS). Table 3 also shows molecular formulae, molecular weight and area percent of major compounds.

\section{Membrane stabilization assay}

MELS at a range of $100 \mu \mathrm{g} / \mathrm{mL}$ to $300 \mu \mathrm{g} / \mathrm{mL}$ prohibits hypotonic lysis of the human erythrocyte membranes. The RBC hemolysis was blocked with the extract at $100 \mu \mathrm{g} / \mathrm{mL}$ at $23.45 \%$ compared with $39.12 \%$ for $100 \mu \mathrm{g} / \mathrm{mL}$ of aspirin. Because the lysosomal membrane components of red human blood cells are similar to each other, the test of anti-inflammatory drug action was the avoidance of HRBC-induced hypotonicity membrane lysis. Results show that MELS can substantially inhibit and change the dose to HRBC hemolysis (Figure 4).

\section{Carrageenan-induced paw edema model}

The MELS (50, 100, $200 \mathrm{mg} / \mathrm{kg}$, p.o.) demonstrated substantial dose-dependent reduction of carrageeninduced rat paw edema from $0.5 \mathrm{hr}$ to $3 \mathrm{hr}$ after

\begin{tabular}{|c|c|c|c|c|c|c|}
\hline $\begin{array}{l}\text { S. } \\
\text { No }\end{array}$ & $\begin{array}{l}\text { Retention } \\
\text { Time }\end{array}$ & Compound name & Canonical Smiles & $\begin{array}{c}\text { Area / } \\
\text { Height (\%) }\end{array}$ & $\begin{array}{c}\text { Molecular } \\
\text { formula }\end{array}$ & $\begin{array}{c}\text { Molecular } \\
\text { weight }\end{array}$ \\
\hline & 11.214 & Morin & $\begin{array}{c}\mathrm{C} 1=\mathrm{CC}(=\mathrm{C}(\mathrm{C}=\mathrm{C} 1 \mathrm{O}) \mathrm{O}) \mathrm{C} 2=\mathrm{C}(\mathrm{C}(=\mathrm{O}) \\
\mathrm{C} 3=\mathrm{C}(\mathrm{C}=\mathrm{C}(\mathrm{C}=\mathrm{C}=3 \mathrm{O} 2) \mathrm{O}) \mathrm{O}) \mathrm{O}\end{array}$ & 2.38 & $\mathrm{C}_{15} \mathrm{H}_{10} \mathrm{O}_{7}$ & 302.23 \\
\hline & 12.575 & Syringic acid & $\operatorname{coc} 1 \mathrm{cc}(\mathrm{C}(\mathrm{O})=\mathrm{O}) \mathrm{cc}(\mathrm{OC}) \mathrm{c} 10$ & 0.49 & $\mathrm{C}_{9} \mathrm{H}_{10} \mathrm{O}_{5}$ & 198.173 \\
\hline & 19.044 & Apigenin & $\mathrm{Oc}(\mathrm{cc} 1) \operatorname{ccc} 1 \mathrm{C}(\mathrm{Oc} 1 \mathrm{c} 2 \mathrm{c}(\mathrm{O}) \mathrm{cc}(\mathrm{O}) \mathrm{c} 1)=\mathrm{CC} 2=\mathrm{O}$ & 0.86 & $\mathrm{C}_{15} \mathrm{H}_{10} \mathrm{O}_{5}$ & 270.239 \\
\hline & 19.335 & Genistic acid. & $\operatorname{CC}(\mathrm{Oc}(\mathrm{cc} 1) \operatorname{cc}(\mathrm{C}(\mathrm{O})=\mathrm{O}) \mathrm{c} 1 \mathrm{OC}(\mathrm{C})=\mathrm{O})=\mathrm{O}$ & 0.48 & $\mathrm{C}_{11} \mathrm{H}_{10} \mathrm{O}_{6}$ & 238.194 \\
\hline & 23.170 & 4-Hydroxy benzoic acid & $\mathrm{OC}(\mathrm{c}(\mathrm{cc} 1) \operatorname{ccc} 10)=\mathrm{O}$ & 0.17 & $\mathrm{C}_{7} \mathrm{H}_{6} \mathrm{O}_{3}$ & 138.122 \\
\hline & 23.407 & 3-Carene & CC1(C)[C@@H]2[C@H]1CC=C(C)C2 & 0.43 & $\mathrm{C}_{10} \mathrm{H}_{16}$ & 136.237 \\
\hline & 24.211 & Camphor & $\mathrm{CC}(\mathrm{C})([\mathrm{C} @ @ \mathrm{H}](\mathrm{CC} 1) \mathrm{C} 2)[\mathrm{C} @ @] 1(\mathrm{C}) \mathrm{C} 2=\mathrm{O}$ & 0.03 & $\mathrm{C}_{10} \mathrm{H}_{16} \mathrm{O}$ & 152.236 \\
\hline & 26.918 & Cinnamic acid & $\mathrm{OC}(/ \mathrm{C}=\mathrm{Cl} 1 \mathrm{ccccc} 1)=\mathrm{O}$ & 0.43 & $\mathrm{C}_{9} \mathrm{H}_{8} \mathrm{O}_{2}$ & 148.161 \\
\hline
\end{tabular}




\begin{tabular}{|c|c|c|c|c|c|c|}
\multicolumn{7}{|c|}{ Table 4: Effect of MELS with carrageenan-induced paw edema in rats. } \\
\hline \multicolumn{1}{|c|}{} & \multicolumn{5}{c|}{ Paw Volume $(\mathbf{m L})$} \\
\hline Groups & Before & $\mathbf{0 ~ h}$ & $\mathbf{0 . 5} \mathbf{~}$ & $\mathbf{1 ~ h}$ & $\mathbf{2} \mathbf{~}$ & $\mathbf{3} \mathbf{~}$ \\
\hline Distilled water & $0.96 \pm 0.02$ & $1.02 \pm 0.02$ & $1.62+0.02$ & $1.89 \pm 0.02$ & $2.01 \pm 0.02$ & $1.95 \pm 0.02$ \\
\hline Diclofenac $20 \mathrm{mg} / \mathrm{kg}$ & $0.98 \pm 0.03$ & $1.05 \pm 0.04$ & $1.42 \pm 0.03^{*}(38.93)$ & $1.54 \pm 0.01^{*}(44.54)$ & $1.59 \pm 0.03^{*}(46.44)$ & $1.44 \pm 0.01^{*}(58.99)$ \\
\hline MELS $50 \mathrm{mg} / \mathrm{kg}$ & $0.96 \pm 0.02$ & $1.04 \pm 0.05$ & $1.58 \pm 0.04(10.6)$ & $1.75 \pm 0.02(19.26)$ & $1.77 \pm 0.02(27.25)$ & $1.68 \pm 0.03(32.11)$ \\
\hline MELS $100 \mathrm{mg} / \mathrm{kg}$ & $0.94 \pm 0.03$ & $1.01 \pm 0.03$ & $1.52 \pm 0.03(15.6)$ & $1.63 \pm 0.01(29.60)$ & $1.64 \pm 0.01(37.35)$ & $1.56 \pm 0.02(41.79)$ \\
\hline MELS $200 \mathrm{mg} / \mathrm{kg}$ & $0.92 \pm 0.04$ & $0.97 \pm 0.04$ & $1.46 \pm 0.03^{*}(18.93)$ & $1.54 \pm 0.03^{*}(35.35)$ & $1.53 \pm 0.02^{*}(44.24)$ & $1.47 \pm 0.01^{*}(47.16)$ \\
\hline
\end{tabular}

Values are presented as the mean $\pm \mathrm{SEM}, n=6$ in each group; values given in parentheses represent PI. One-way ANOVA followed by multiple Tukey's comparison test. $* p<0.05$, as compared to the control group.

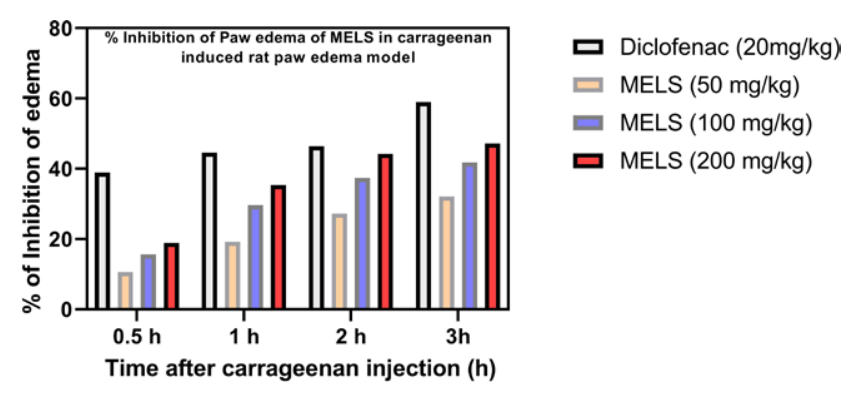

Figure 5: Inhibition of carrageenan-induced rat paw edema by Diclofenac and MELS measured at $0.5,1,2$ and $3 \mathrm{~h}$ after carrageenan injection.

drug administration relative to control group. MELS recorded average PI of paw edema at concentrations of $50,100,200 \mathrm{mg} / \mathrm{kg}$ p.o. as $18.93,35.35,44.24$ and $47.16(P<0.05)$ (Table 4, Figure 5).

\section{Molecular Docking Studies}

In order to identify a prospective candidate for managing antioxidant and anti-inflammatory activity, molecular docking was executed over nine phytoconstituents acquired from L. spinosa on the binding pocket of targets. Regarding in-silico antioxidant activity, Morin, apigenin and Genistic acid. Displayed best docking score, among the phytochemicals. Rest of the compounds i.e., 3-Carene. 4-hydroxy benzoic acid, Alpha pinene, Camphor, Cinnamic acid and Syringic acid also interacted well with targets showing moderate binding energies (Tables 5-10 and Figures 6-10).

Total of 3 compounds was selected based on the binding interactions with $5 \mathrm{IKR}$. Out of the three compounds, Morin exhibited the best-docked score $(-9.3 \mathrm{Kcal} / \mathrm{mol})$ with COX-2(5IKR), followed by apigenin and Genistic acid. With binding affinity of -9.1 and $-6.8 \mathrm{kcal} / \mathrm{mol}$.

\section{Drug likeliness}

The physicochemical properties of the chosen nine active compounds were studied on DruLiTo software.

\begin{tabular}{|c|c|c|c|c|c|}
\hline \multirow{2}{*}{$\begin{array}{l}\text { Sr. } \\
\text { No }\end{array}$} & \multirow{2}{*}{ Drug } & \multicolumn{4}{|c|}{ Dock Score (Kcal/mol) } \\
\hline & & $10 G 5$ & $1 N 8 Q$ & 1DNU & $3 N R Z$ \\
\hline 1 & 3-Carene & -5.6 & -5.6 & -5.2 & -5.7 \\
\hline 2 & 4-hydroxy benzoic acid & -5.9 & -6.2 & -6.1 & -7 \\
\hline 3 & Alpha pinene & -5.5 & -5.8 & -4.9 & -5.4 \\
\hline 4 & Apigenin & -8.5 & -8.1 & -9.8 & -7.8 \\
\hline 5 & Camphor & -5.8 & -5.8 & -5 & -6.1 \\
\hline 6 & Cinnamic acid & -6 & -5.8 & -7.4 & -6.1 \\
\hline 7 & Gensticacid & -6.8 & -6.3 & -7.2 & -6.2 \\
\hline 8 & Morin & -8.5 & -8.2 & -9.8 & -8.2 \\
\hline 9 & Syringic acid & -6 & -5.8 & -6.8 & -5.6 \\
\hline
\end{tabular}

Table 6: Interactions of Cytochrome P450 (PDB ID:
10G5) amino acid residues with ligands at receptor
sites.


Table 7: Interactions of lypoxygenase (PDB ID: 1N8Q) amino acid residues with ligands at receptor sites

\begin{tabular}{|c|c|c|c|c|}
\hline \multirow[b]{2}{*}{ Ligands } & \multirow{2}{*}{$\begin{array}{l}\text { Binding Affinity, } \\
\Delta G(\text { Kcal/mol) }\end{array}$} & \multicolumn{3}{|c|}{ Amino acids involved and Distance $\left(A^{\circ}\right)$} \\
\hline & & Hydrogen Binding Interactions & $\begin{array}{l}\text { Hydrophobic } \\
\text { Interactions }\end{array}$ & $\begin{array}{l}\text { Electrostatic } \\
\text { Interactions }\end{array}$ \\
\hline Apigenin & -8.1 & $\begin{array}{c}\text { ARG A:200 (3.47, 4.01), ASP A:158 } \\
\text { (4.79), GLY A:189 (4.22) }\end{array}$ & ILE A:201 (5.45) & - \\
\hline Gensticacid & -6.3 & $\begin{array}{c}\text { ARG A:200 }(4.75,4.09,3.44), \text { ASN } \\
\text { A:186 (4.90) }\end{array}$ & - & - \\
\hline Morin & -8.2 & $\begin{array}{c}\text { VAL A:256 (5.02), THR A:274 (3.79), } \\
\text { TYR A:275 (5.00) }\end{array}$ & $\begin{array}{c}\text { LEU A:258 (6.54), ALA } \\
\text { A:263 (4.87), PHE A:272 } \\
(6.45)\end{array}$ & ARG A:260 $(5.39,7.42)$ \\
\hline Syringic acid & -5.8 & ARG A:200 (4.80), SER A:157 (4.11) & LYS A:156 (4.88) & - \\
\hline
\end{tabular}

\begin{tabular}{|c|c|c|c|c|}
\hline \multirow[b]{2}{*}{ Ligands } & \multirow{2}{*}{$\begin{array}{c}\text { Binding Affinity, } \\
\Delta \mathbf{G}(\mathrm{Kcal} / \mathrm{mol})\end{array}$} & \multicolumn{3}{|c|}{ Amino acids involved and Distance $\left(A^{\circ}\right)$} \\
\hline & & $\begin{array}{l}\text { Hydrogen Binding } \\
\text { Interactions }\end{array}$ & Hydrophobic Interactions & $\begin{array}{l}\text { Electrostatic } \\
\text { Interactions }\end{array}$ \\
\hline Apigenin & -7.8 & $\begin{array}{c}\text { ILE C:1229 (3.50)GLU A:45 } \\
(4.89)\end{array}$ & $\begin{array}{l}\text { LEU A:147 (6.38), ALA C:1231 (6.39), } \\
\text { GLY A:46 (5.65), ALA B:338 (4.84) }\end{array}$ & LYS C:1228 $(5.22,6.56)$ \\
\hline Gensticacid & -6.2 & $\begin{array}{l}\text { SER A:69 (3.44), LYS B:340 } \\
\text { (3.84), LYS B:343 (4.52) }\end{array}$ & LYS B:340 (4.11) & - \\
\hline Morin & -8.2 & ILE C:1229 (3.61) & $\begin{array}{c}\text { ALA C:1231 }(4.91,4.99,6.67), \text { LEU } \\
\text { A:147 }(6.02)\end{array}$ & LYS C:1228 $(5.44,6.08)$ \\
\hline
\end{tabular}

\begin{tabular}{|c|c|c|c|c|}
\hline \multirow[b]{2}{*}{ Ligands } & \multirow{2}{*}{$\begin{array}{c}\text { Binding } \\
\text { Affinity, } \Delta G \\
\text { (Kcal/mol) }\end{array}$} & \multicolumn{3}{|c|}{ Amino acids involved and Distance $\left(\mathrm{A}^{\circ}\right)$} \\
\hline & & $\begin{array}{l}\text { Hydrogen Binding } \\
\text { Interactions }\end{array}$ & Hydrophobic Interactions & Electrostatic Interactions \\
\hline Apigenin & -9.8 & $\begin{array}{c}\text { GLY A:335 (3.51), ARG C:333 } \\
\text { (4.08), ASP A:98 (4.93), GLN } \\
\text { A:91 (5.18) }\end{array}$ & $\begin{array}{c}\text { GLY A:90 (6.00), ARG A:333 } \\
(5.13)\end{array}$ & $\begin{array}{c}\text { HIS A:95 (6.40), HIS C:336 } \\
\text { (5.54), MET A:87 (7.45), ASP } \\
\text { A:94 }(5.28,5.72)\end{array}$ \\
\hline Gensticacid & -7.2 & $\begin{array}{c}\text { HIS A:95 (5.52), THR C:329 } \\
\text { (4.36), ARG C:333 (3.85), ASP } \\
\text { A:94 (5.06) }\end{array}$ & $\begin{array}{c}\text { ARG C:239 (4.05), ARG C:333 } \\
(4.97)\end{array}$ & $\begin{array}{c}\text { ASP A:94 (5.32), HIS C:336 } \\
(5.03)\end{array}$ \\
\hline Morin & -9.8 & $\begin{array}{c}\text { THR C:329 (3.59), ARG A:333 } \\
\text { (4.23), PHE C:332 (3.66), MET } \\
\text { A:87 (3.44) }\end{array}$ & GLY A:90 (5.98) & $\begin{array}{c}\text { ARG C:239 (6.55), ASP A:94 } \\
(6.11,5.41), \text { HIS C:336 (5.36) } \\
\text { HIS A:95 (6.60) }\end{array}$ \\
\hline
\end{tabular}

\section{Table 10: Interactions of xanthine COX-2 (PDB ID: 5IKR) amino acid residues with ligands at receptor sites}

\begin{tabular}{|c|c|c|c|}
\hline \multirow{2}{*}{ Ligands } & \multirow{2}{*}{$\begin{array}{l}\text { Binding Affinity, } \\
\Delta G(\text { Kcal/mol) }\end{array}$} & \multicolumn{2}{|c|}{ Amino acids involved and Distance $\left(\mathrm{A}^{\circ}\right)$} \\
\hline & & Hydrogen Binding Interactions & Hydrophobic Interactions \\
\hline Apigenin & -9.1 & $\begin{array}{c}\text { CYS A:47 (3.39, 4.29), CYS A:36 (2.88), GLN } \\
\text { A:461 (5.28) }\end{array}$ & $\begin{array}{c}\text { LEU A:152 (5.27), PRO A:153 (4.91, 6.52), } \\
\text { CYS A:36 (6.46), ARG A:469 (6.84), VAL } \\
\text { A:46 (3.66) }\end{array}$ \\
\hline Genistic acid & -6.8 & ASN A:43 (4.58), GLN A:461 (5.88) & $\begin{array}{c}\text { ARG A:44 (3.54), LEU A:152 (5.51), PRO } \\
\text { A:153 (4.90) }\end{array}$ \\
\hline Morin & -9.3 & CYS A:41 (2.99), GLY A:135 (4.99) & $\begin{array}{c}\text { CYS A:36 (6.41), ARG A:44 (4.16), LEU } \\
\text { A:152 (5.79), PRO A:153 }(4.78,5.05)\end{array}$ \\
\hline
\end{tabular}




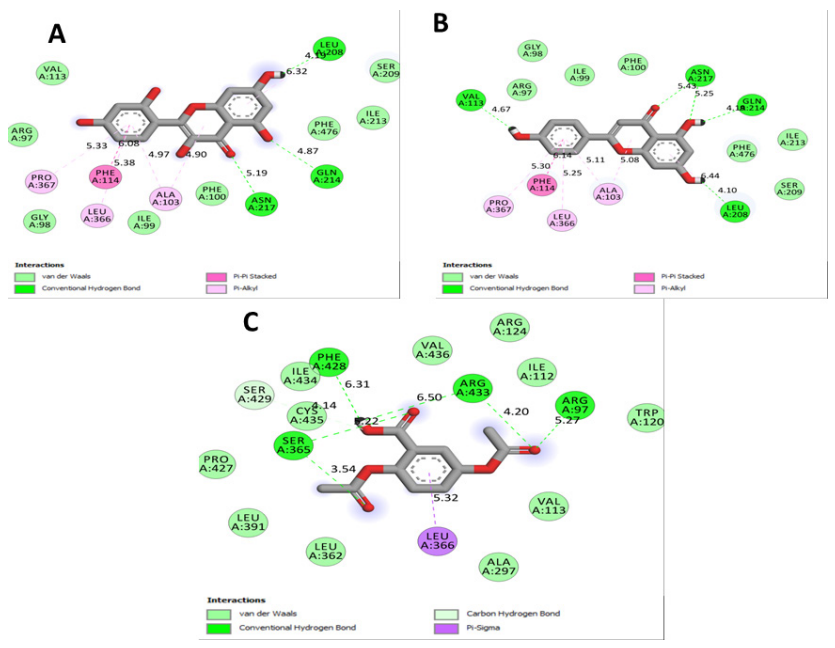

Figure 6: Various Two-dimensional Interactions of ligands with cytochrome P450 (1PG5). A: Morin; B: Apigenin; C: Genistic acid.

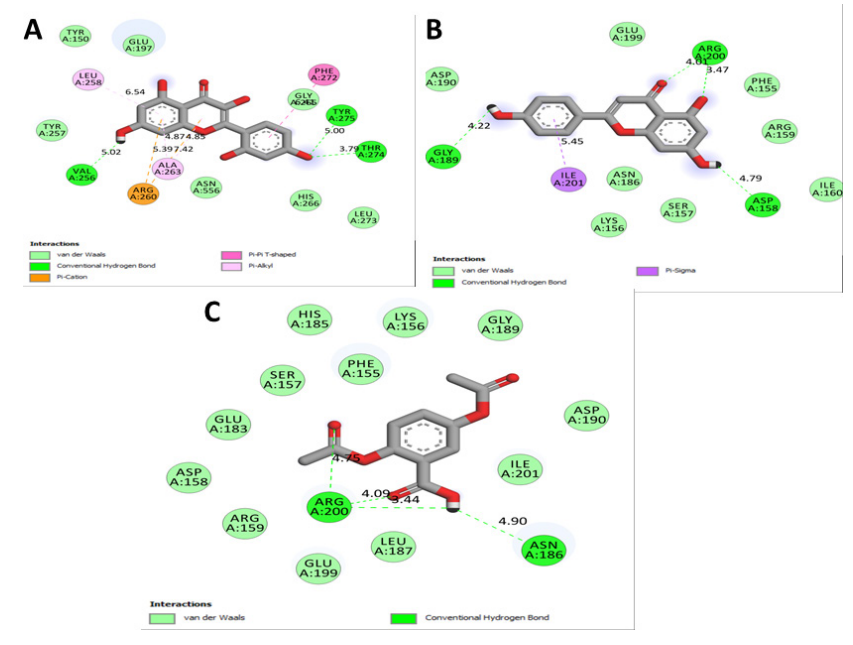

Figure 7: Various Two-dimensional Interactions of ligands with lypoxygenase (1NBQ). A: Morin; B: Apigenin; C: Genistic acid.

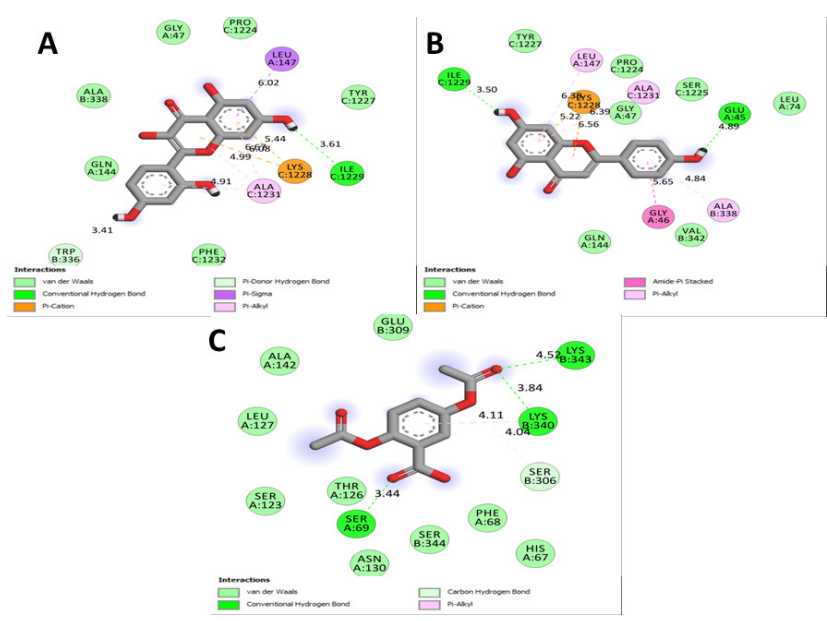

Figure 8: Various Two-dimensional Interactions of ligands with myeloperoxidase (3NRZ). A: Morin; B: Apigenin; C: Genistic acid.

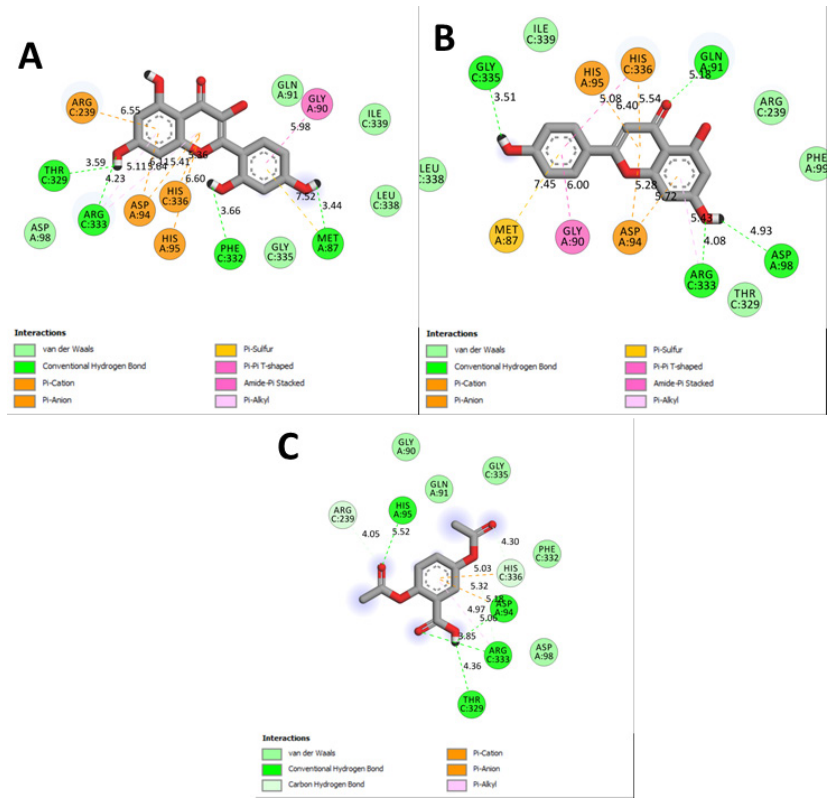

Figure 9: Various Two-dimensional Interactions of ligands with xanthine oxidase (1DNU). A: Morin; B: Apigenin; C: Genistic acid.

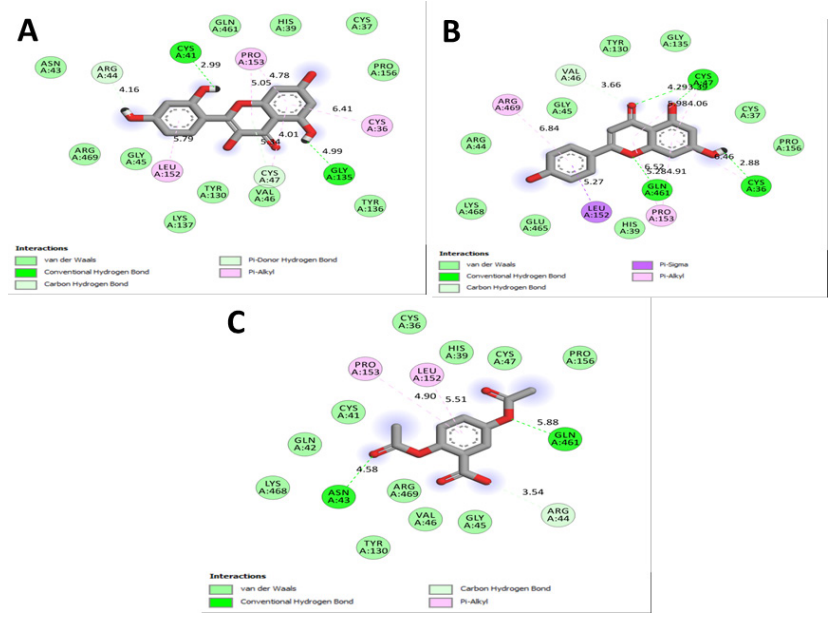

Figure 10: Various Two-dimensional Interactions of ligands with Cyclo oxygenase-2 (5IKR). A: Morin; B: Apigenin; C: Genistic acid.

As all the compounds are of natural source, none of them obeyed Lipinski's rule (Table 11).

\section{ADME/T evaluation by using admetSAR}

The ADMET properties of the ligands were assessed, making use of admetSAR. ADMET properties for the substances in the research study were evaluated, making use of admetSAR. All the substances revealed excellent human intestinal absorption (HIA), blood-brain barrier (B.B.B.) infiltration. None of the compound was found carcinogenic. All the compounds were AMES negative. 
Table 11: Physicochemical properties of the active compounds and accordance with the rules of drug-likeness

\begin{tabular}{|c|c|c|c|c|c|c|c|c|c|c|}
\hline Sr. No. & Compound & MW & logp & Alogp & HBA & HBD & TPSA & AMR & $\begin{array}{c}\text { nRB } \\
\text { Violations }\end{array}$ \\
\hline 1 & Morin & 291.96 & 1.405 & -1.244 & 7 & 0 & 26.3 & 83.44 & 1 & 0 \\
\hline 2 & Genstic acid & 227.97 & 0.569 & -0.088 & 6 & 0 & 69.67 & 59.04 & 5 & 0 \\
\hline 3 & $\begin{array}{c}4-\text { hydroxy } \\
\text { benzoic acid }\end{array}$ & 131.98 & 0.268 & 0.405 & 3 & 0 & 17.07 & 38.56 & 1 & 0 \\
\hline 4 & Cinnamic acid & 139.99 & 1.887 & 1.329 & 2 & 0 & 17.07 & 47.2 & 2 & 0 \\
\hline 5 & Syringic acid & 187.97 & 0.748 & -0.592 & 5 & 0 & 35.53 & 51.85 & 3 & 0 \\
\hline 6 & Apigenin & 259.97 & 1.138 & -0.224 & 5 & 0 & 26.3 & 80.15 & 1 & 0 \\
\hline 7 & Alpha-pinene & 120 & 4.177 & 1.516 & 0 & 0 & 0 & 44.54 & 0 & 0 \\
\hline 8 & 3-Carene & 120 & 4.181 & 2.143 & 0 & 0 & 0 & 44.99 & 0 & 0 \\
\hline 9 & Camphor & 135.99 & 2.127 & 0.969 & 1 & 0 & 17.07 & 43.76 & 0 & 0 \\
\hline
\end{tabular}

\begin{tabular}{|c|c|c|c|c|c|}
\hline \multicolumn{7}{|c|}{ Table 12: ADME/T Properties of different compounds from L. spinus. } \\
\hline Ligands & HIA & BBB & AMES Toxicity & Carcinogenicity & LD $_{50}$ in rat (mol/kg) \\
\hline Morin & 0.9855 & 0.6286 & Non AMES Toxic & Non-carcinogen & 3.0825 \\
\hline Genstic acid & 0.9541 & 0.9335 & Non AMES Toxic & Non-carcinogen & 2.7396 \\
\hline 4-hydroxy benzoic acid & 0.9872 & 0.532 & Non AMES Toxic & Non-carcinogen & 1.3983 \\
\hline Cinnamic acid & 0.9945 & 0.9526 & Non AMES Toxic & Non-carcinogen & 1.7416 \\
\hline Syringic acid & 0.9165 & 0.5861 & Non AMES Toxic & Non-carcinogen & 2.5353 \\
\hline Apigenin & 0.9887 & 0.6364 & Non AMES Toxic & Non-carcinogen & 2.6983 \\
\hline Alpha-pinene & 0.9964 & 0.8959 & Non AMES Toxic & Non-carcinogen & 1.5348 \\
\hline 3-Carene & 0.9943 & 0.9473 & Non AMES Toxic & Non-carcinogen & 1.4843 \\
\hline Camphor & 0.9971 & 0.9836 & Non AMES Toxic & Non-carcinogen & 1.6328 \\
\hline
\end{tabular}

The results of HIA, B.B.B., $\mathrm{LD}_{50}$ values for the compounds are listed in Table 12.

\section{DISCUSSION}

Oxygen is essential for survival but under certain abnormal physiological conditions converts some of oxygen to ROS by univalent reactions that makes them highly reactive and damages nucleic acids, proteins, lipids and carbohydrates components of cell. ${ }^{14}$ Such freely available radicals control essential aspects of cell biology such as signals, gene transcriptions and controls on soluble guanylate cyclase activities. ${ }^{15}$ The excess production of free radicals results in abnormal physiological conditions like oxidative stress that impedes various cellular and metabolic functions leading to neuro-degenerative diseases, gastroduodenal disorders, cancer, cataracts, premature ageing, inflammation, cardiovascular diseases, ${ }^{16}$ Chronic kidney disease ${ }^{17}$ and metabolic dysfunction. ${ }^{18}$ Naturally and synthetically antioxidant activity has been linked to different processes such as chain initiation reduction, binding metal ion transition catalysts, peroxide decomposition, ongoing abstract hydrogen avoidance and radical scavenging ${ }^{16,19}$ that hamper consequences of oxidative damage. Phytochemicals like flavonoids, phenols, tannins and related polyphenols ${ }^{20}$ are potential natural antioxidants with strong antioxidant capacity that offers protection against oxidative deterioration by the radicals. ${ }^{14}$

$\mathrm{H}_{2} \mathrm{O}_{2}$ has an substantial physiologic significance in which the intracellular levels of $\mathrm{Ca}^{2+}$ increase, transcription factors are triggered, certain genes are repressed, cell proliferation facilitated or blocked, certain pathways for signal transduction triggered or disrupted and apoptosis facilitated or undermined. ${ }^{14}$ During certain adverse physicochemical, environmental or pathological conditions the level of $\mathrm{H}_{2} \mathrm{O}_{2}$ increases that yields potent species $\mathrm{OH}$, highly reactive free radical in biological system. ${ }^{20,21}$ They negatively influence lipid, proteins and DNA, in particular thiamine and guanosine and are implicated in other diseases such as cardiovascular 
disorders, cytotoxicity and ageing. ${ }^{17,22}$ The scavenging of $\mathrm{H}_{2} \mathrm{O}_{2}$ by the extract may be attributed to active secondary metabolites, phenolics which neutralize $\mathrm{H}_{2} \mathrm{O}_{2}$ by donating electrons thereby neutralizing it to water. ${ }^{23}$ MELS 'scavenging capability was attributed to phytochemicals' structural specificity, which dictates their ability to donate electron. MELS demonstrated high dose-dependent $\mathrm{H}_{2} \mathrm{O}_{2}$ scavenging activity. This split may be triggered by direct reactions to $\mathrm{H}_{2} \mathrm{O}_{2}$, reaction to $\mathrm{H}_{2} \mathrm{O}_{2}$ intermediates and inhibiting horseradish peroxidase from $\mathrm{H}_{2} \mathrm{O}_{2}$ binding. ${ }^{24}$

Numbers of epidemiological studies have highlighted the inverse correlation between antioxidants and occurrence of disease and mortality due to these diseases. ${ }^{20}$ Phenolic compounds are secondary synthesis products with high redox effects, which may play an significant role in intermediate metals, lipoxygenase inhibition and non-scavenging radicals. ${ }^{25}$ Preliminary phytochemical investigation revealed that root part of $L$. spinosa is rich in secondary metabolites. ${ }^{26}$ The presence of phytochemicals, phenolic and polyphenolic constituents might be responsible for the free radical scavenging activities in the present experiment. Polyphenolic compounds, rich in antioxidants, can inhibit human mutagenesis and carcinogenesis ${ }^{27}$ which also involves stabilizing lipid peroxidation. ${ }^{20}$

Since an animal model for extreme swelling, carrageeninduced edema remains largely to be used and is actually considered biphasic. The initial (1-2 h) is largely solved in cell-ruined environments by histamine, serotonin and increased prostaglandin formation. The latter stage (3h) is liable to release prostaglandin and regulated by tissue macrophages, bradykinin, leukotriens. ${ }^{28,29}$ MELS's latestage, substantial suppressive activity $(P<0.05)$ indicates its powerful anti-inflammatory effect. It is comparable to diclofenac, which avoided edema at $10 \mathrm{mg} / \mathrm{kg}$ by $61.44 \%$. Statistically important finding $(P<0.05)$. Ueno et al. (2000) reported that rodent paw carragein therapy results in bradykinine production that eventually leads to prostaglandin biosynthesis, as well as many other autacoids that accumulate inflammatory exudates..$^{30,31}$ PGE2 is a dominant vasodilator with many endogenous vasodilators, notably histamine and bradykinine, in severe inflammatory environments. Extract action mode is firmly recommended to suppress prostaglandin synthesis. Tests revealed that MELS has essential antiinflammatory properties at various stages. Carrageenininduced inflammation is an essential pathway to determine anti-inflammatory function. Oedema formation in rat paw following carrageen injection stems from histamine, serotonin and prostaglandin release and associated substances. MELR's good anti-inflammatory behavior. ${ }^{12,32,33}$ Due to the anabolic steroids, flavonoids and glycosides present in the extract, this significant anti-inflammatory and even analgesic impact that result from the obstacle of any kind of inflamed negotiators. Latest results indicate $L$. spinosa efficacy in treating acute inflammation. Additionally, the end result authenticates the mythology-relevant anti-inflammatory information as well as the L. spinosa extracts.

Out of 9 candidates, three compounds displayed a higher binding affinity and least binding energy with the all the targets responsible for antioxidant and antiinflammatory activity. Morin has exhibited highest dock score with least binding energy of -8.5, -8.2, -9.8, -8.2 and $-9.3 \mathrm{Kcal} / \mathrm{mol}$ with $1 \mathrm{OG} 5,1 \mathrm{~N} 8 \mathrm{Q}, 1 \mathrm{DNU}, 3 \mathrm{NRZ}$ and $5 \mathrm{IKR}$.

In silico measurement of intestinal absorption, both phytochemicals were theoretically strongly soluble in the gastrointestinal tract (Table 5). Despite effective distribution of medications in the human body, significant toxic effects such as carcinogenicity became important concerns. Accordingly, in the early phases of drug growth, identification and evaluation of novel product candidates is proposed to be important. No compounds demonstrated in silico toxicity in this analysis.

\section{CONCLUSION}

Thus the findings of present investigation support the traditional ethanomedicinal claims of $L$. spinosa for the treatment of diverse infections. The antioxidant activities of $L$. spinosa can be used as a natural antioxidant or nutraceuticals and might be effective to diminish oxidative stress associated with different pathophysiological conditions. Within this analysis, the bioactive phytocompounds for drug production and testing have been identified. This combinatorial analytic procedure can be beneficial to develop phyto compounds as novel candidates for the treatment of inflammatory disorders by antioxidant and anti-inflammatory action in invitro and invivo. Such a plant screening may also provide a source of new bioactive compounds with functional characteristics that help restore health.

\section{ACKNOWLEDGEMENT}

The authors were thankful to A.U. College of Pharmaceutical Sciences, Andhra University for providing necessary laboratory facilities to carry out present research work.

\section{CONFLICT OF INTEREST}

The authors declare that there is no conflict of interest. 


\section{ABBREVIATIONS}

HIA: Human Intestinal absorption; B.B.B: Blood Brain Barrier; $\mathbf{L D}_{50}$ : Lethal dose 50\%; MELR: Methanolic extract of Lasia spinosa; $\mathbf{H}_{2} \mathbf{O}_{2}$ : Hydrogen peroxide; DPPH: 2, 2-diphenyl-1-picrylhydrazyl; MW: Molecular Weight; HBA: Hydrogen bond acceptors; HBD: Hydrogen bond Donors; TPSA: Total Protein Surface area; AMR: Molar refractivity; nRB: No. of rotatable bonds.

\section{REFERENCES}

1. Pendota S, Ndhlala A, Aremu A, Aderogba M, Staden JV. Anti-inflammatory, antioxidant and in silico studies of Buddleja salviifolia (L). Lam leaf constituents. South African Journal of Botany. 2014;93:79-85.

2. Ashraf $Z$, Alamgeer MK, Hassan M, Abdullah $S$, Waheed $M$, Ahsan $H$, et al. Flurbiprofen-antioxidant mutual prodrugs as safer nonsteroidal anti-inflammatory drugs: Synthesis, pharmacological investigation and computational molecular modeling. Drug Design, Development and Therapy. 2016;10:2401.

3. Amaravani M, Prasad NK, Ramakrishna V. COX-2 structural analysis and docking studies with gallic acid structural analogues. Springer Plus. 2012;1(1):58.

4. Cao H, Yu R, Choi Y, Ma ZZ, Zhang H, Xiang W, et al. Discovery of cyclooxygenase inhibitors from medicinal plants used to treat inflammation. Pharmacological Research. 2010;61(6):519-24.

5. Kumar S. Leafy and edible plants of North-East India: Scientific Publishers. 2003

6. Patra JK, Das G, Kumar S, Thatoi H. Ethnopharmacology and Biodiversity of Medicinal Plants: CRC Press. 2019.

7. Gokhale MS, Kokate C. Practical pharmacognosy: Editora Record. 2008.

8. Khandelwal KR. Practical pharmacognosy: Pragati Books Pvt. Ltd. 2008.

9. Gordon MH, Paiva-Martins F, Almeida M. Antioxidant activity of hydroxytyrosol acetate compared with that of other olive oil polyphenols. Journal of Agricultural and Food Chemistry. 2001;49(5):2480-5.

10. James $\mathrm{O}$, Nnacheta $\mathrm{O}$, Wara $\mathrm{H}$, Aliyu $\mathrm{U}$. In vitro and in vivo studies on the antioxidative activities, membrane stabilization and cytotoxicity of water spinach (Ipomoea aquatica Forsk) from Ibaji Ponds, Nigeria. International Journal of PharmTech Research. 2009;1(3):474-82.

11. Joseph J, Bindhu AR, Aleykutty NA. In vitro and in vivo Antiinflammatory Activity of Clerodendrum paniculatum Linn. Leaves. Indian J Pharm Sci. 2013;75(3):376-9.

12. Winter CA, Risley EA, Nuss GW. Carrageenin-induced edema in hind paw of the rat as an assay for antiinflammatory drugs. Proceedings of the Society for Experimental Biology and Medicine. 1962;111(3):544-7.

13. Praveen K, Yellamma K. In silco identification of suitable acetuylcholinesterase inhibitors from Morinda citrifolia linn. with reference to alzheimer's disease. International Journal of Pharmaceutical Sciences and Research. 2014;5(12):5474.

14. Prakash D, Gupta K. The antioxidant phytochemicals of nutraceutical importance. The Open Nutraceuticals Journal. 2009;2(1).

15. Fang $Y Z$, Yang $S$, Wu G. Free radicals, antioxidants and nutrition. Nutrition. 2002;18(10):872-9.
16. Bahorun $\mathrm{T}$, Soobrattee $\mathrm{M}$, Luximon-Ramma V, Aruoma O. Free radicals and antioxidants in cardiovascular health and disease. Internet Journal of Medical Update. 2006;1(2):25-41.

17. Small DM, Coombes JS, Bennett N, Johnson DW, Gobe GC. Oxidative stress, anti-oxidant therapies and chronic kidney disease. Nephrology. 2012;17(4):311-21.

18. López-Alarcón $\mathrm{C}$, Denicola A. Evaluating the antioxidant capacity of natural products: A review on chemical and cellular-based assays. Analytica Chimica Acta. 2013;763:1-10. DOI.

19. Gülçin I, Küfrevioğlu Öi, Oktay M, Büyükokuroğlu ME. Antioxidant, antimicrobial, antiulcer and analgesic activities of nettle (Urtica dioica L.). Journal of Ethnopharmacology. 2004;90(2-3):205-15.

20. Devasagayam T, Tilak J, Boloor K, Sane KS, Ghaskadbi SS, Lele R. Free radicals and antioxidants in human health: Current status and future prospects. Japi. 2004;52(794804):4

21. Singh S, Singh R. In vitro methods of assay of antioxidants: An overview. Food Reviews International. 2008;24(4):392-415.

22. Badole SL, Zanwar AA, Khopade AN, Bodhankar SL. In vitro antioxidant and antimicrobial activity cycloart-23-ene-3ß,-25-diol (B2) isolated from Pongamia pinnata (L. Pierre). Asian Pacific Journal of Tropical Medicine. 2011;4(11):910-6.

23. Mathew $\mathrm{S}$, Abraham TE. In vitro antioxidant activity and scavenging effects of Cinnamomum verum leaf extract assayed by different methodologies. Food and Chemical Toxicology. 2006;44(2):198-206.

24. Huang $D$, Ou B, Prior RL. The chemistry behind antioxidant capacity assays Journal of Agricultural and Food Chemistry. 2005;53(6):1841-56.

25. Mohamed AA, Ali SI, El-Baz FK. Antioxidant and antibacterial activities of crude extracts and essential oils of Syzygium cumini leaves. Plos One. 2013;8(4).

26. Suresh H, Shivakumar B, Shivakumar S. Inhibitory potential of the ethanol extract of Annona reticulata Linn. against melanoma tumor. Journal of Natural Pharmaceuticals. 2011;2(4).

27. Amir M, Mujeeb M, Khan A, Ashraf K, Sharma D, Aqil M. Phytochemical analysis and in vitro antioxidant activity of Uncaria gambir. International Journal of Green Pharmacy. 2012;6(1).

28. Chavan M, Wakte $P$, Shinde D. Analgesic and anti-inflammatory activities of 18-acetoxy-ent-kaur-16-ene from Annona squamosa L. bark. Inflammopharmacology. 2011;19(2):111-5.

29. DeOliveira MJ, Truiti MDCT, Muscara MN, Bolonheis SM, Dantas JA, Caparroz-Assef SM, et al. Anti-inflammatory activity of crude extract and fractions of Nectandra falcifolia leaves. Biological and Pharmaceutical Bulletin. 2006;29(11):2241-5.

30. Ueno A, Naraba H, Ikeda Y, Ushikubi F, Murata T, Narumiya S, et al. Intrinsic prostacyclin contributes to exudation induced by bradykinin or carrageenin: A study on the paw edema induced in IP-receptor-deficient mice. Life Sciences. 2000;66(12):PL155-60.

31. Simplice $\mathrm{FH}$, Armand AB, Roger P, Emmanuel AA, Pierre K, Veronica $\mathrm{N}$. Effects of Hibiscus asper leaves extracts on carrageenan induced oedema and complete Freund's adjuvant-induced arthritis in rats. Journal of Cell and Animal Biology. 2011;5(5):69-75.

32. Georgewill O, Georgewill U, Nwankwoala R. Anti-inflammatory effects of Morninga oleifera lam extract in rats. Asian Pacific Journal of Tropical Medicine. 2010;3(2):133-5

33. Georgewill $\mathrm{O}$, Georgewill U. Evaluation of the anti-inflammatory activity of extract of Vernonia amygdalina. Asian Pacific Journal of Tropical Medicine. 2010;3(2):150-1. 
PICTORIAL ABSTRACT

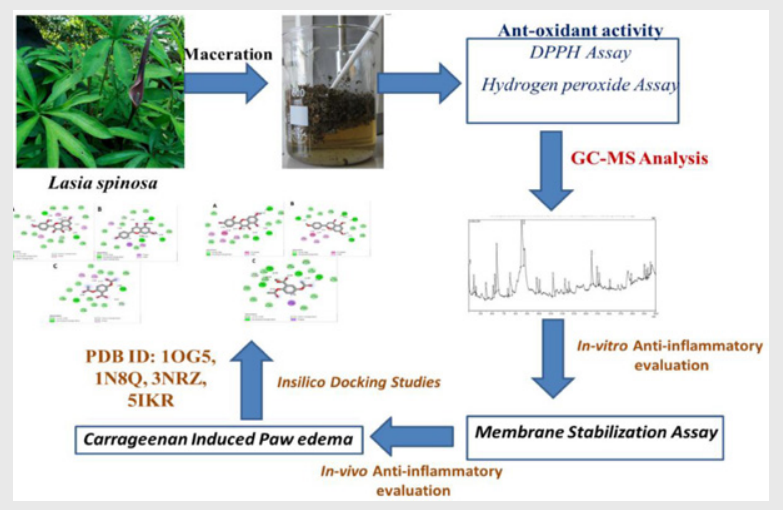

\section{SUMMARY}

- The methanolic extract of Lasia spinosa has significant antioxidant (invitro) and antiinflammatory activity (invitro and invivo) in comparison with other extract.

- The methanolic extract was subjected to GC-MS analysis, showed the presence of Morin,

- Syringic acid; Apigenin, Genistic acid, 4-Hydroxy benzoic acid, 3-Carene, Camphor, Cinnamic acid.

- Amongst the isolated compounds, Morin has potent efficacy as an antioxidant and anti-inflammatory activity, with a best docking score in comparison with other constituents.

- All the isolated compounds have no toxicity and are non-carcinogenic in nature.

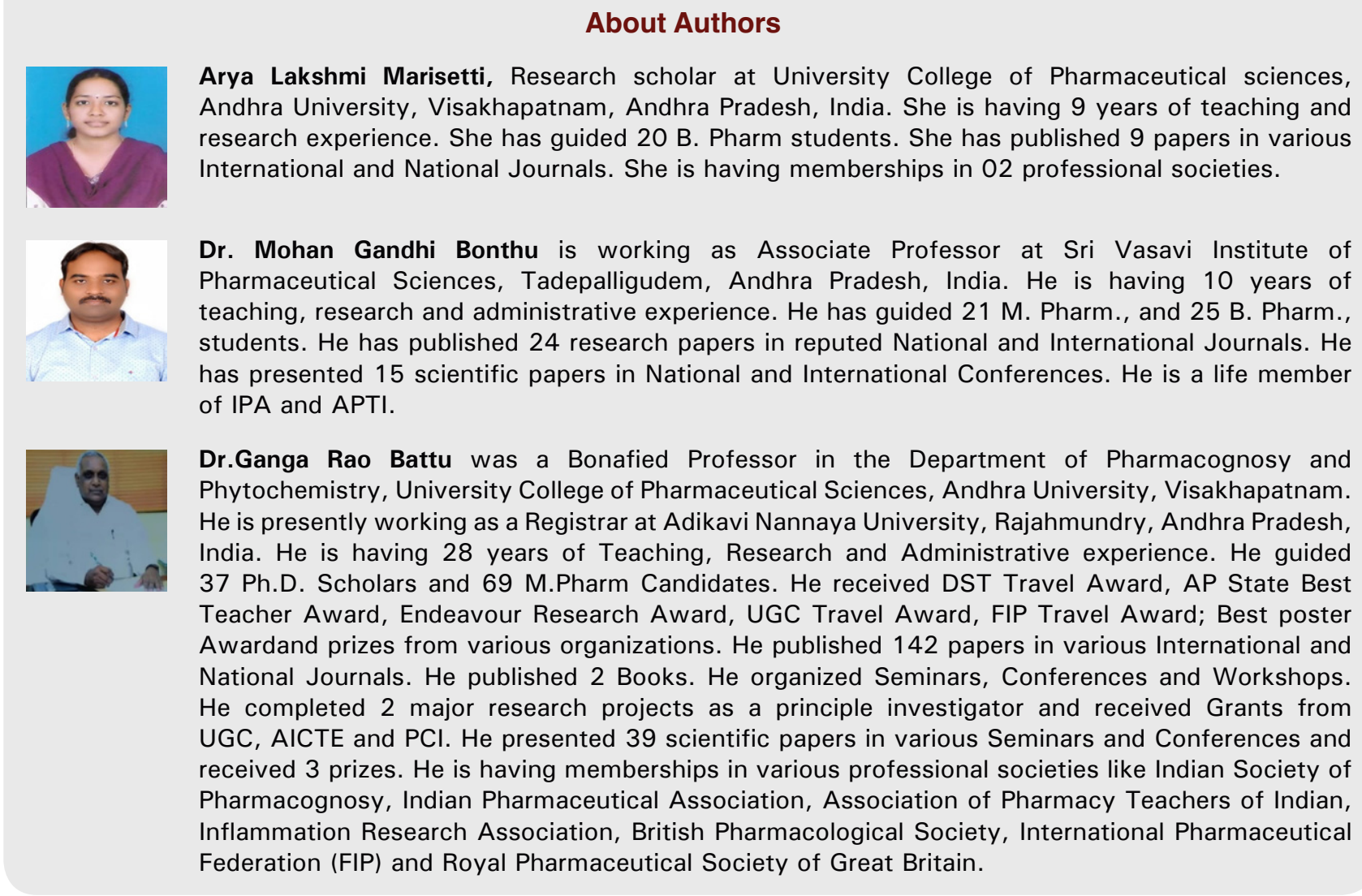

Cite this article: Marisetti AL, Bonthu MG, Battu GR. Anti-oxidant and Anti-inflammatory Activity Screening of Lasia spinosa Rhizome and its Validation using a Computational Simulation Approach. Indian J of Pharmaceutical Education and Research. 2020;54(4):1109-20. 